\title{
Research on Optimizing Region-based Clustering for LEACH Routing Protocol of WSN
}

\author{
Zhang Zhongxian*, Gu Yali and Yu Bing
}

Department of Computer Engineering, Zhangzhou Institute of Technology, Zhangzhou, Fujian, 363000, P.R. China

\begin{abstract}
This paper intends to improve the current LEACH Hierarchical Routing Protocol of WSN, in order to solve the problems of uneven distribution of cluster heads and the imbalanced energy consumption caused by randomly selecting the cluster heads. This paper proposes an algorithm of optimizing the number of the cluster heads, which divides the cluster regions evenly according to the minimum distance of the cluster heads which are determined by the optimal number of cluster heads. Furthermore, our novel approach restricts the cluster heads rotation within the cluster region, with our optimized algorithm, so it can guarantee an optimal balanced point for each interval of the cluster rotations, which saves the network energy consumption, and prolongs the generation period of the network, and improves the load balance of the network as well. Therefore, our approach improves the network performance of WSN significantly.
\end{abstract}

Keywords: Cluster region optimization, cluster-head distribution, hierarchical routing, LEACH, WSN.

\section{INTRODUCTION}

Considering the topology, Wireless Sensor Network (WSN) routing protocol can be roughly divided into two categories: flat routing protocols and hierarchical routing protocols [1]. Among them, the hierarchical routing protocols, due to the good scalability and the ability of optimized management on the energy-balance for the network node. LEACH [2] is the first hierarchical routing protocol of WSN, which uses a hierarchical dynamic routing technology to equilibrium energy-load to the entire network nodes by selecting cluster-heads periodically and randomly.It not only reduces the network energy-consumption but also extends the survival time of the network. Furthermore, it improves the scalability and robustness of the network by the collaboration of node blocks. However, LEACH protocol selects a random node as a cluster-head, which may cause the uneven distribution and the imbalanced energy-consumption of the cluster-heads. LEACH-C [3] is a modified LEACH routing protocol, which introduces the mechanisms of the base station control, so the nodes can report their location and energy information periodically to the base station. It also solves the problem of choosing the best amounts and optimal locations for the cluster-heads by using the simulated annealing algorithm. On the other hand, due to reporting the information of the nodes periodically, LEACH-C will increase the nodes energy-consumption and the network traffic, concurrently, the time delay and signal-interference will be caused as well. Then [4] (Threshold Sanative Energy Efficient Sensor Network Protocol) is another modified LEACH protocol, which is a sensor network protocol based on the threshold-energy. It uses the similar cluster structure and functions of LEACH protocol, and has features of strong real-time, but it's easy to cause the signal-interference, and is suitable for the environment of continuous data-collection. HEED [5] (Hybrid Energy-Efficient Distributed clustering) is to improve the problem of uneven distribution of clusterheads of the LEACH protocol. Its characteristic is that the cluster-heads are fully distributed, so the cluster-heads distribution and energy-consumption is uniform, though, it causes the larger network delay. PEGASIS [6] (PowerEfficient Gathering in Sensor Information System) is also an improvement based on LEACH protocol. It has improved the problem of node balance a bit; however, it has a long time delay and poor real-time performance.

In LEACH protocol, the number of cluster-heads and their distribution has a great impact on the entire network. If the number of cluster-heads is too small, the distance of the data-transmission and loads of data-processing of clusterheads will be increasing. If cluster-heads are too many, it will lead increases of the total energy- consumption of each rotation of the cluster-heads, at the same time, the reduce of data-fusion efficiency will cause unnecessary energyconsumption. The fact that LEACH randomly generates cluster-heads is likely to cause the uneven distribution of cluster-heads, also may make little energy nodes be elected as cluster-heads. This paper proposes a novel routing protocol based on LEACH, which optimizes the creation of cluster region, selection of cluster-heads, as well as the decision of the number and the distribution of region clusters.

\section{PROTOCOL OPTIMIZATION ALGORITHM}

\subsection{Design Ideas}

(1) According to the principle of minimum energy consumption, LEACH protocol should have an optimal number of cluster-heads [7], so the entire network can be divided into regions whose amount will correspond to the number of cluster-heads. 


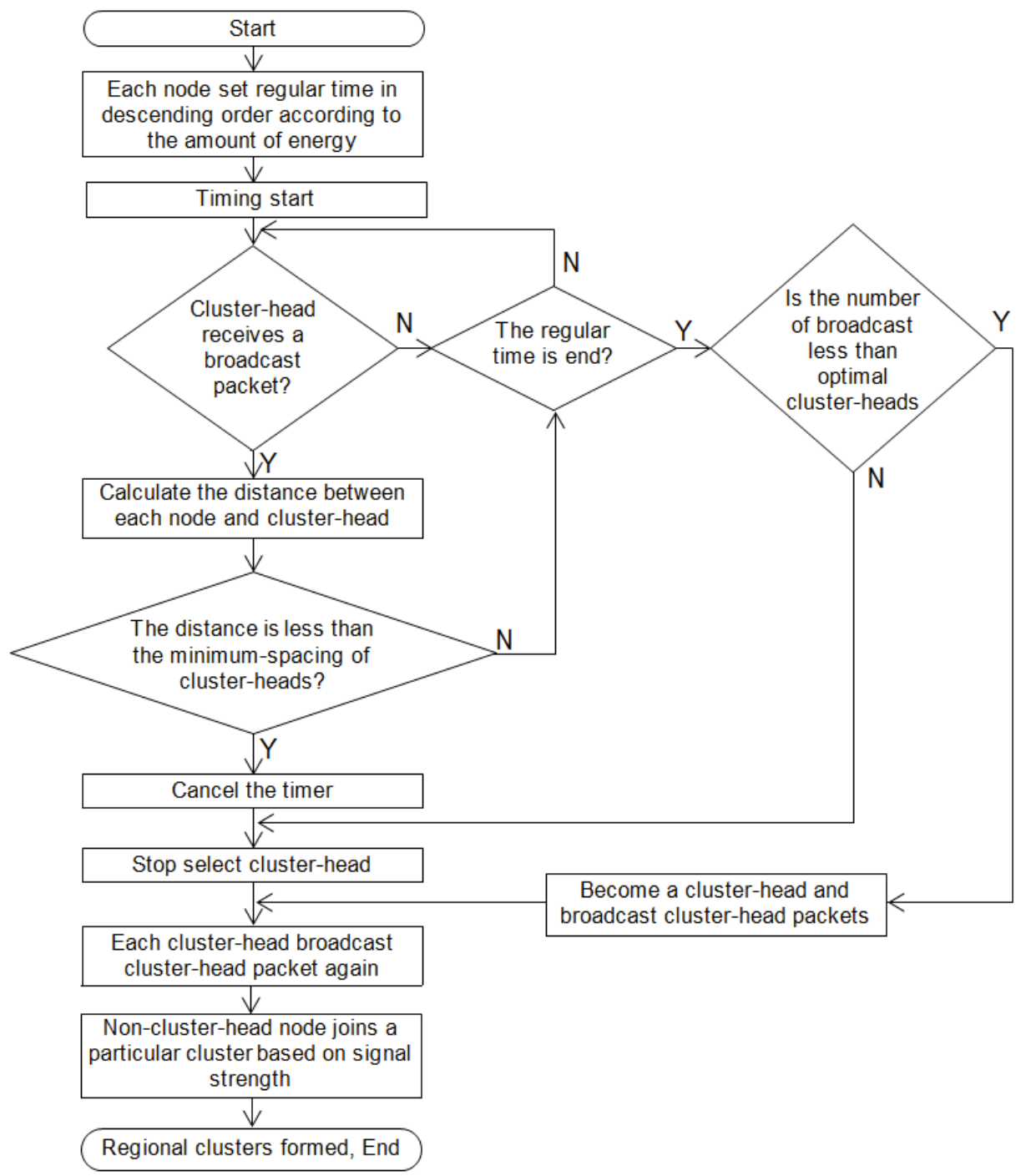

Fig. (1). Algorithm flowchart for regional clusters forming.

(2) The minimal distance between the cluster-heads can be determined by the optimal number of the cluster-heads. So the selection for the first cluster-head can be implemented, then a balanced distribution of cluster-heads will be achieved as well.

(3) After the first cluster-head selection is completed, a balanced distributed cluster region can be created, corresponding to each cluster-head. In addition, the rotation of the cluster-head for each node can be carried out and limited in the same cluster region.

(4) In order to save the network energy -consumption and extend the life cycle, the interval between the rotations of cluster-head should have an optimal balance point. (5) For each round of the selection of the cluster-head in the cluster region, the node with most energy left will be selected as the cluster-head.

\subsection{The Formation of Cluster Regions}

The algorithm of forming cluster regions is shown in Fig. (1).
(1) Each node that has been identified as the cluster-head will broadcast the cluster-head information packet to inform the other nodes.

(2) Each node maintains a timer, whose starting value is associated with the remaining energy of the node. The more energy the node remains, the shorter the time left. Apparently, the timer for the node with most energy left in the network will be set at first so that the node will be the first cluster-head in the network.

(3) Once the other nodes receive the broadcast packets from the cluster-head, their distance with the cluster-head will be calculated immediately. If the distance is shorter than the minimal interval, the timer will be interrupted and the operation for selecting cluster-head will be cancelled immediately, otherwise, the operation will continue until the timer runs out.

(4) Once the timer runs out, if the number of clusterheads hasn't reached the optimal number yet, the nodes will become cluster-heads randomly, until the optimal number is satisfied, to complete the selection of network cluster-heads. 
(5) Each cluster-head broadcasts the information packet with its own identification, so the other non-head nodes can join an appropriate cluster according to the signal intensity. Then the cluster region partitions complete.

\subsection{Cluster-Head Rotation Intra Cluster Region}

The algorithm of cluster-head rotation intra cluster region is shown in Fig. (2).

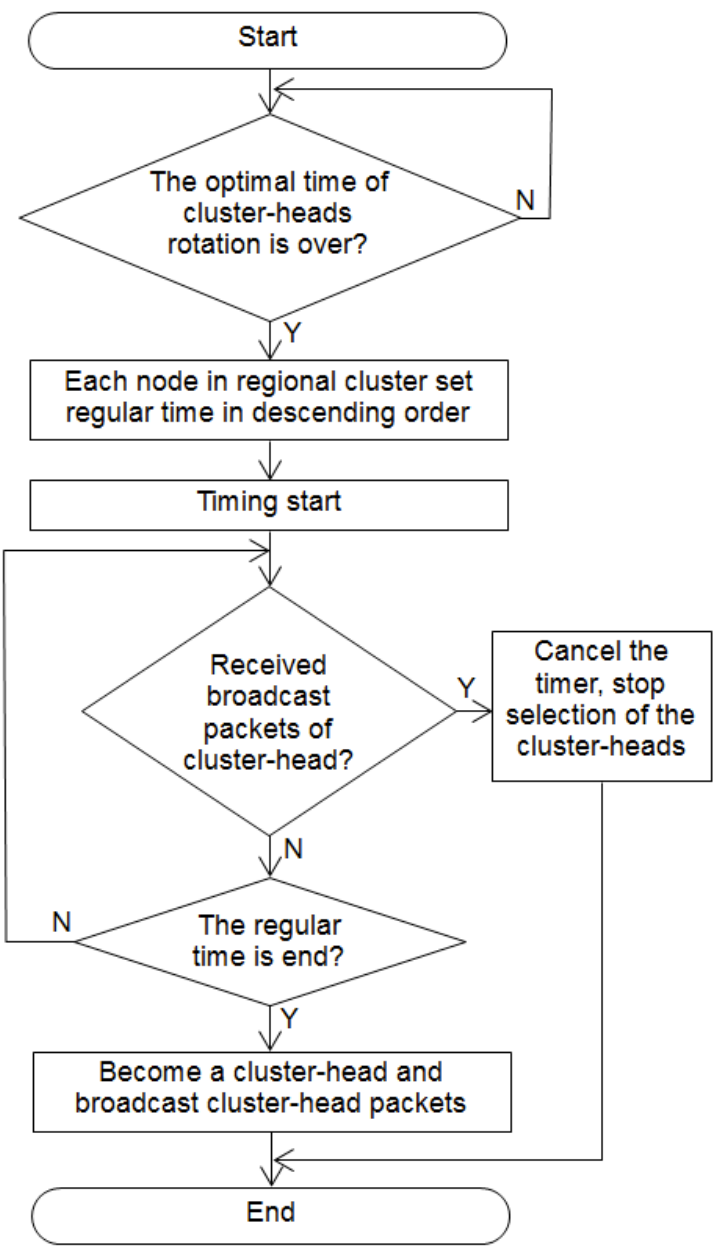

Fig. (2). Algorithm flowcharts for cluster-head rotation in regional cluster.

Once the optimal rotation time interval of the clusterhead is reached, the timer for the intra cluster nodes will be set in descending order in accordance with the energy and start timing

The timer for the node which has the most energy will first run out and become the cluster-head of the intra cluster region, and broadcast the packet and inform the other intra cluster nodes to cancel their timers.

\section{THE PARAMETER OPTIMIZATION ALGORITHM}

\subsection{The Optimal Number of Cluster Regions}

The optimal number of cluster regions is determined in accordance with the criterion of minimum energy consumption throughout the network. Network energy consumption includes the consumption of the cluster-head and the total consumption of the other member nodes intra the cluster regions. According to the communication energy mode of LEACH protocol [8], cluster-head node energy consumption mainly includes three aspects: receiving data from member nodes, data fusion and data transmission. In addition, according to the distance of transmitting data from the node, cluster-head node energy should attenuate following the multi-path channel model (i.e. $\mathrm{d} 4$ attenuation); and the member nodes energy should attenuate following the free space channel model (i.e. $\mathrm{d} 2$ attenuation).

Let a square area $S * S$ be divided into $m$ regions, and $n$ nodes be randomly distributed. We can define:

$E_{c h}=L\left(E_{e}(n / m-1)+n E_{c m i x} / m+\varepsilon_{r} d_{c}^{4}\right)$

Where $E_{c h}$ is the energy -consumption of each cluster node; $L$ is the frame length of each information data; $E_{e}$ is the energy -consumption for receiving circuit; $E_{c m i x}$ is the fusion energy -consumption of the cluster node; $\boldsymbol{\varepsilon}_{r}$ is the energy -consumption factor of multi-path channel model power amplification; $d_{c}$ is the distance between the clusterhead node and the base station.

Let $\varepsilon_{f}$ be the energy -consumption factor of the free space channel model power amplification; let $d_{m e m}$ be the distance between the member node and the cluster-head, we can define the energy -consumption of each member node as follows

$$
E_{m e m}=L\left(E_{e}+\varepsilon_{f} d_{m e m}\right)
$$

The network nodes are randomly distributed in the region with the density $\rho(r, \theta)$, so the expected value of the square of the distance from member nodes to the cluster-head $E$ $\left(d_{\text {mem }}^{2}\right)$ is:

$$
E\left(d_{m e m}^{2}\right)=\iint r^{2} \rho(r, \theta) d r d \theta
$$

The cluster region is a circular area with the radius of $S / \sqrt{\pi m}$. If the nodes are evenly distributed within the cluster region, let's say $\rho=1 / S^{2} \mathrm{~m}$, then the above equation can be expressed as:

$E\left(\mathrm{~d}_{\mathrm{mem}}^{2}\right)=\rho \int_{\theta=0}^{2 \pi} \int_{r=0}^{\frac{S}{\sqrt{\pi m}}} r^{3} d r d \theta=\frac{\rho S^{4}}{2 \pi m^{2}}=\frac{1}{2 \pi} \frac{S^{2}}{m}$

Therefore, the energy of the member nodes can be defined as followed:

$E_{m e m}=L\left(E^{e}+\varepsilon_{f} \cdot \frac{1}{2 \pi} \frac{S^{2}}{m}\right)$

The total energy of the whole network $E_{\text {all }}$ can be defined:

$$
\begin{aligned}
& E_{\text {all }}=m\left(E_{c h}+E_{\text {mem }}\right) \\
& \approx L\left(2 n E_{e}+n E_{c \text { mix }}+m \varepsilon_{r} d_{c}{ }^{4}+n \varepsilon_{f} \frac{1}{2 \pi} \frac{S^{2}}{m}\right)
\end{aligned}
$$

So we can get the best number $m_{\text {opt }}$ of clusters region by computing the minimum value of $E_{\text {all }}$ : 


$$
m_{\text {opt }}=\frac{\sqrt{n}}{\sqrt{2 \pi}} \sqrt{\frac{\varepsilon_{f}}{\varepsilon_{r}}} \frac{S}{d_{c}^{2}}
$$

\subsection{The Minimum Distance D Between the Cluster Heads}

Once the best number is confirmed, the minimum distance $D$ between the cluster-heads will be easily to compute from:

$$
D=S / \sqrt{m^{o p} t}=\sqrt{\frac{\sqrt{n}}{\sqrt{2 \pi}}} \sqrt{\frac{\varepsilon_{f}}{\varepsilon_{r}}} \frac{S}{d_{c}^{2}}
$$

\subsection{The Time Interval of the Cluster-head Rotation}

The optimal number of the cluster-head rotation should satisfy that, each node from the cluster region could be the cluster-head once and also be the member node for every other node being the cluster-head, in other words, each node can be the cluster-head for once and the member node for $\mathrm{n} / \mathrm{m}-1$ times. Suppose, for each round, the average number for the data frame of the cluster-head rotation is $n_{f / r}$, the energy -consumption of the cluster-head is $E_{c h / r}$, the energy consumption of the member node is $E_{m e m / r}$. Then:

$$
\begin{aligned}
& E_{c h / r}=n_{f / r} E_{c h} \\
& E_{m e m / r}=n_{f / r} E_{m e m}
\end{aligned}
$$

Let $E_{\text {init }}$ be the initial energy of each node be; let $R$ be the bit rate of the transmit data. By combining the formula (2-1), (2-5), we can obtain the time interval for each round of cluster-head rotation as follows,

$$
t_{c h / r}=\frac{n}{m} \frac{1}{R} \frac{E_{\text {init }}}{\left(E_{e} \frac{n}{m}+E_{c m i x} \frac{n}{m}+\varepsilon_{r} d_{c}^{4}\right)+\left(\frac{n}{m}-1\right)\left(E_{e}+\varepsilon f \frac{1}{2 \pi} \frac{S^{2}}{m}\right)}
$$

\section{SIMULATION AND RESULT ANALYSIS}

Network lifetime and the total amount of the data received by the base station are the key indicators to evaluate the energy efficiency and validity of the WSN router protocol and the reliability of the network. In addition, our algorithm focuses on improving the cluster optimization for the current LEACH protocol, so the analysis of the cluster and the load of the cluster-head should also be considered. Therefore, to evaluate the algorithm performance, we consider all the three indicators that have been mentioned above, which include the network lifetime, total amount of the data reception and the load balance factor (LBF).

\subsection{Simulation Environment and Initial Parameters Set- ting}

Assume 100 sensor nodes are randomly distributed in a $100 \mathrm{~m} * 100 \mathrm{~m}$ square area, and the coordinates of the base station are $(50,175)$, the parameters that are related to the formulas from section 3.1 could be initialized as follows:

The transmitting data bit rate is $R=1 \mathrm{Mb} / \mathrm{s}$; the energy consumption of receiving circuit is $E_{e}=50 \mathrm{~nJ} / \mathrm{bit}$; the energy - consumption for data fusion of cluster nodes is $E_{\text {cmix }}=$ $5 \mathrm{~nJ} /$ bit; the initial energy of node is $E_{\text {init }}=2 \mathrm{~J}$; the length of information data packet is $L=500 * 8$ bit; the factor of the energy-consumption for power of the multi-path channel is $\boldsymbol{\varepsilon}_{r}=0.013(\mathrm{pJ} / \mathrm{bit}) / \mathrm{m}^{2}$; the factor of the energy -consumption for power of the free space channel is $\varepsilon_{f}=10(\mathrm{pJ} / \mathrm{bit}) / \mathrm{m} 2$.

Let the range of the number of the cluster-heads be 1 to 7 , then from Formula (2-11), we can get $t_{c h / r}=16.7 \sim 17.8 \mathrm{~s}$. Taking the time for selecting the cluster-heads into account, we set the time interval of cluster-head rotation as $20 \mathrm{~s}$, for simplicity. Also from Formula (2-11), we can infer that the optimal number of the cluster regions is 4 .

The following section will show the results of our simulation experiments, which have been run on the platform of Windows7+Cygwin+NS-2.33, for simulating the classic LEACH, LEACH-C and our improved protocol. Meanwhile, the relevant analysis of our simulation results will be discussed.

\subsection{Performance Analysis of Simulation Result}

Fig. (3) shows the comparison of the lifetime of the network nodes for the three protocols according to our simulation results. The lifetime of the first node for the classic LEACH, LEACH-C, and our improved protocol is $230 \mathrm{~s}$, 280 s and 310 s, respectively. Furthermore, the lifetime of the last node for the three protocols is respectively 530s, 590s and $620 \mathrm{~s}$. Obviously, the longest survival time of the network nodes is based on our improved protocol that has been proposed in this paper. In conclusion, our improved protocol increased the network survival time by $20 \%$ compared to the classic protocol LEACH.

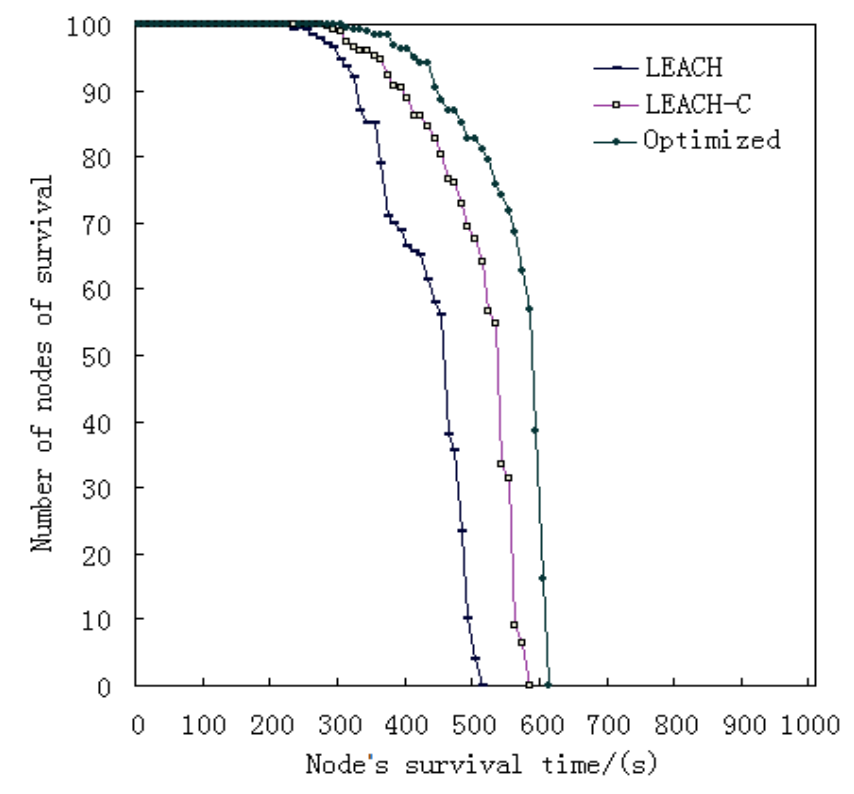

Fig. (3). The simulation results of the survival time of the network nodes for three classic protocols.

Fig. (4) shows the comparison of the amount of data that received from the base station of the three classic protocols according to our simulation results. The performance of the amount of the data is consistent with the simulation results of the network lifetime. For the classic LEACH, LEACH-C, 


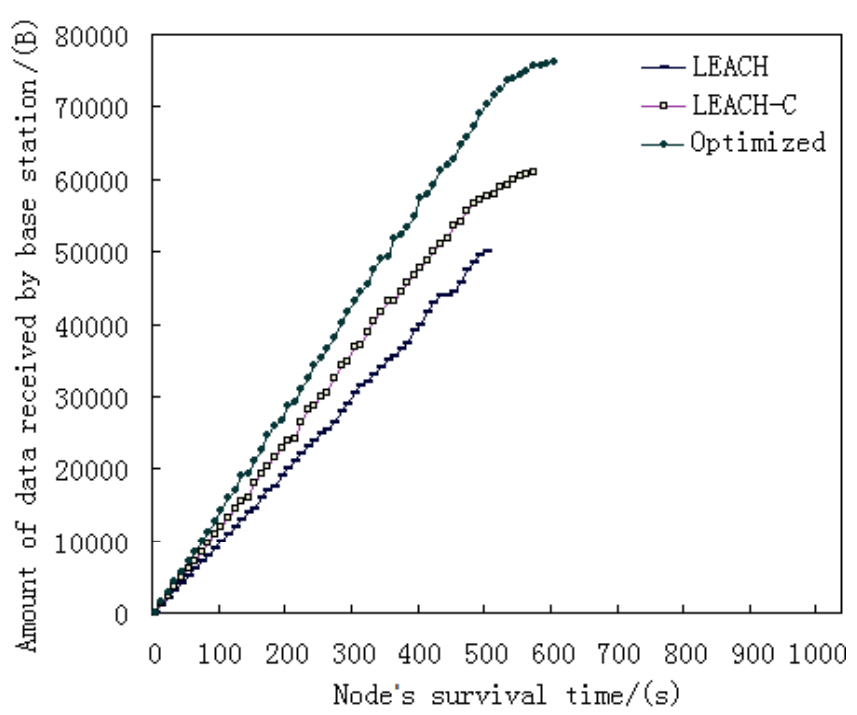

Fig. (4). The simulation results of amount of data received by base station for three classic protocols.

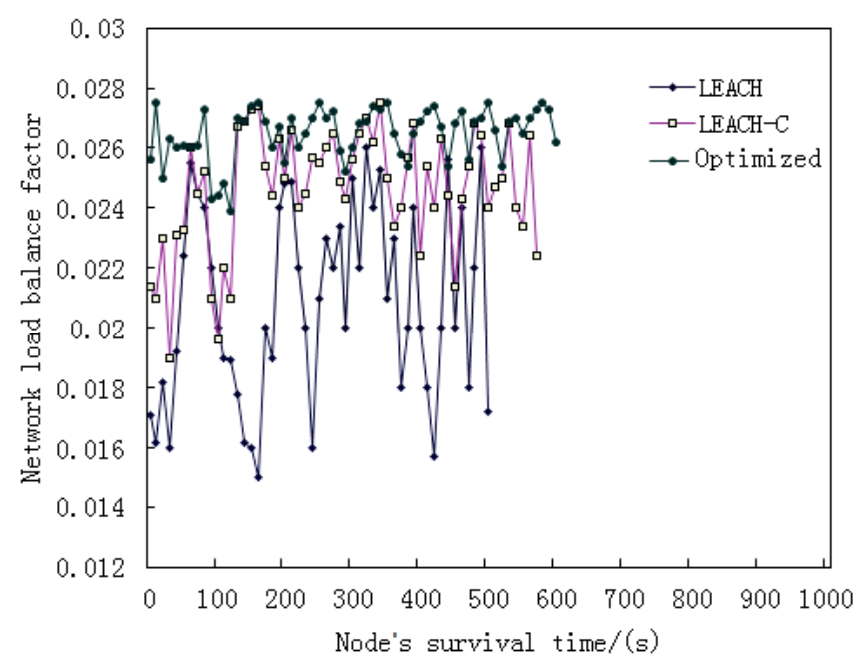

Fig. (5). The simulation results of network load balance factor for three classic protocols.

and our improved protocol, the amount of data received by the base stations stops increasing at 530s, 590s and $620 \mathrm{~s}$ respectively, that is to say, it reaches its maximum. Obviously, the largest amount of data received by the base station is based on our improved protocol; then the LEACH-C; LEACH has the lowest amount. This shows that the improved protocol that has been proposed in this paper increased the amount of data received by the base station by $45 \%$, compared to the classic LEACH protocol.

Fig. (5) shows the comparison of network load balance factor for the three protocols, according to our simulation results. Consistently, least fluctuation is caused by the network load balance factor from our improved protocol. Thus it can be seen that, our improved protocol's network load balance factor performs the best; LEACH-C is the suboptimal one, the classic protocol LEACH is the worst of the three protocols from our simulation experiments.

\section{CONCLUSION}

This paper presents a framework for improving the classic LEACH and LEACH-C WSN protocol. We consider dividing the cluster regions by solving the optimal number of the cluster-heads. In addition, the processing of the clusterhead rotation is restricted internally, where the optimal balance point of the rotation time interval has been ensured. Furthermore, we propose to partition the cluster region evenly in accordance with the minimum distance between the cluster-heads. According to the discussion and simulation experiments we did, our proposed protocol has significantly improved the performance of the network, which includes the WSN network energy efficiency and validity, the network reliability and the network load equilibrium etc. Compare to the classic LEACH and even other improvements of LEACH.

\section{CONFLICT OF INTEREST}

The authors confirm that this article content has no conflict of interest.

\section{ACKNOWLEDGEMENTS}

Declared none.

\section{REFERENCES}

[1] F.Y. Ren, H. Huang, and C. Lin, "Wireless sensor network," Journal of Software, vol. 14, pp. 1282-1291, 2003.

[2] W. Heinemann, A. Chandrakasan, and H. Balakrishnan, "Energyefficient communication protocol for wireless micro-sensor networks," Proceedings of the $33^{\text {rd }}$ IEEE Annual Hawaii International Conference on System Sciences, Maui, 2000, pp. 3005-3014.

[3] W. Heinemann, A. Chandrakasan, and H. Balakrishnan, "Application-specific protocol architectures for wireless networks," IEEE Transactions on Wireless Communications, vol. 1, pp. 660-670, 2002.

[4] A. Manjeshwar, and D.P. Grawal, "TEEN:A protocol for enhanced efficiency in wireless sensor network," Proceedings of the $1^{\text {st }}$ International Workshop on Parallel and Distributed Computing Issues in Wireless Network and Mobile Computing, 2001, pp. 2009-2015.

[5] O. Younis, S. Fahmy, and A. Hybird, "Energy-efficient, distributed clustering approach for Ad-hoc sensor network," IEEE Transactions On Mobile Computing, vol. 3, pp. 660-669, 2004.

[6] S. Lindsey, and C.S. Raghavendra, "PEGASIS: power-efficient gathering in sensor information system," Proceedings of the IEEE Aerospace Conference, Aerospace and Electronic Systems Society, Montana, 2002, pp. 1125-1130.

[7] R. Yan, S. Zhang, and H. Zhang, "Three-dimensional optimal coverage routing protocol in wireless sensor networks," Acta Electronica Sinica, vol. 34, pp. 306-311, February. 2006.

[8] H. Y. Zhou, J. Xu, Y. Gao, H. P. Xie, D. C. Zuo, J. Li, and P. Zhou, "Research and design on energy model of sensor node based on state transition," Application Research of Computers, vol. 29, pp. 432-436, September, 2012.

\footnotetext{
Received: September 16, 2014

Revised: December 23, 2014

Accepted: December 31, 2014

(C) Zhongxian et al.; Licensee Bentham Open.
}

This is an open access article licensed under the terms of the Creative Commons Attribution Non-Commercial License (http://creativecommons.org/licenses/by-nc/3.0/) which permits unrestricted, non-commercial use, distribution and reproduction in any medium, provided the work is properly cited. 